\title{
Clinicopathological features of metaplastic breast carcinoma
}

\author{
Oğuz Ahmet Hasdemir ${ }^{1, A-F}$, Serhat Tokgöz, ${ }^{1, B-D, F}$, Fulya Köybaşıoğlu, $2, B, C, E$, \\ Harun Karabacak ${ }^{1, C D}$, Cüneyt Yücesoy ${ }^{3, B, F}$, Gökşen İnanç İmamoğl L $^{4, B, F}$ \\ ${ }^{1}$ Department of General Surgery, Ministry of Health Dışkapı Yıldırım Beyazıt Training and Research Hospital, Ankara, Turkey \\ ${ }^{2}$ Department of Pathology, Ministry of Health Dışkapı Yıldırım Beyazıt Training and Research Hospital, Ankara, Turkey \\ ${ }^{3}$ Department of Radiology, Ministry of Health Dışkapı Yıldırım Beyazıt Training and Research Hospital, Ankara, Turkey \\ ${ }^{4}$ Department of Medical Oncology, Ministry of Health Dışkapı Yıldırım Beyazıt Training and Research Hospital, Ankara, Turkey \\ A - research concept and design; B - collection and/or assembly of data; C - data analysis and interpretation; \\ $D$ - writing the article; $E$ - critical revision of the article; $F$ - final approval of the article
}

\author{
Address for correspondence \\ Oğuz Hasdemir \\ E-mail: oguzhasdemir@gmail.com \\ Funding sources \\ None declared \\ Conflict of interest \\ None declared
}

Received on October 9, 2016

Reviewed on November 1, 2016

Accepted on January 5, 2017

DOI

$10.17219 /$ acem $/ 68293$

\section{Copyright}

Copyright by Author(s)

This is an article distributed under the terms of the

Creative Commons Attribution Non-Commercial License

(http://creativecommons.org/licenses/by-nc-nd/4.0/)

\begin{abstract}
Background. Metaplastic carcinoma of the breast $(\mathrm{MpBC})$ is defined as a group of heterogeneous malignant neoplasms that contain glandular and non-glandular components with mixed epithelial and mesenchymal differentiations.
\end{abstract}

Objectives. The aim of this study was to research the clinical and pathological characteristics of $\mathrm{MpBC}$ determining its rank among all breast cancers.

Material and methods. Metaplastic carcinoma of the breast was found in 7 out of 1,164 patients who had been diagnosed with breast cancer within the period of 12 years in our hospital. Demographic and clinical characteristics of the patients were retrieved from the patient files, and their final status was verified by a phone call. Diagnoses of the patients were confirmed by examining hematohylen and eosin (H\&E) preparations. They were stained immunohistochemically for estrogen receptor (ER), progesterone receptor (PR), (-erbB-2, CK5/6 (Sitokeratin5/6), and EGFR (epidermal growth factor receptor), and the subgroups were determined according to the WHO classification.

Results. All patients were female with a median age of 61 years (41-87 years). Three of them were diagnosed with stage IIB, 2 with IIIB and 1 with IV. Four patients had squamous type of metaplastic cell differentiation, 1 spindle, 1 adenosquamous, and 1 osteosarcomatous. In 6 out of 7 patients, ER, PR and C-erbB-2 expressions were negative immunohistochemically. In the case of squamous metaplasia, estrogen receptor was $10 \%$ and progesterone receptor was 5\% positive. CK5/6 was positive in 5 cases. Epidermal growth factor was positive in all cases.

Conclusions. Metaplastic carcinoma of the breast is relatively rare and, in our series, its incidence was $0.6 \%$. According to its immunohistochemical characteristics, $\mathrm{MpBC}$ can be interpreted as a subgroup of triplenegative breast cancers (TNBC). Five of the presented patients resembled the subgroup of TNBC with a basaloid phenotype. The chemotherapy regimens suggested in the treatment of $\mathrm{MpBC}$ are platin in the epithelial subgroup and high-dose anthracycline in the mesenchymal subgroup. There is a need of new studies that evaluate different choices of treatment as MpBC has a bad prognosis and an aggressive nature.

Key words: metaplastic breast cancer, breast cancer, triple-negative breast cancer 


\section{Introduction}

Breast cancer is the most frequent cancer in women. Metaplastic carcinoma of the breast $(\mathrm{MpBC})$ is a very rare and aggressive subgroup with a bad prognosis within the breast cancers. ${ }^{1-3}$ Metaplastic carcinoma of the breast is defined as a group of heterogeneous malignant neoplasms that contain glandular and non-glandular components with mixed epithelial and mesenchymal differentiations. ${ }^{4}$ Estrogen receptor (ER), progesterone receptor (PR) and human epidermal growth factor receptor 2 (HER2/neu, c-erbB-2) are negative in more than $90 \%$ of $\mathrm{MpBC}$, while cytokeratin $5 / 6$ (CK5/6), cytokeratin 14, and epidermal growth factor receptor (EGFR) show expressions immunohistochemically. ${ }^{5}$ This subgroup, for which standard treatment protocols have different responses due to its heterogeneous nature, needs new and tumor-specific treatment methods.

In this study, the research of the clinical and pathologic characteristics of the $\mathrm{MpBC}$ that determine its rank among all breast cancers was aimed.

\section{Methods}

Metaplastic carcinoma of the breast was found in 7 out of 1,164 patients that had a diagnosis of breast cancer within the period of 12 years in our hospital. Demographic and clinical characteristics of the patients were retrieved from the patient files, and their final status was verified by a phone call. Histopathological findings of the patients were evaluated by examining the preparations fixed in formalin and stained with hematoxylen and eosin (H\&E).

They were immunochistochemically re-evaluated for ER (1/100, clone EP-1), PR (1/400, SP2), C-erbB-2 (1/800, e24001+3B5), CK5/6 (1/100, D5/16B4), and EGFR (1/25, EP38Y). Hormone receptors, nuclear staining, and the reaction intensity of the tumor cells were evaluated immunohistochemically. The staining percentage of the tumor cell nuclei was noted. Estrogen receptors and PR were considered positive if the nuclear staining was more than $10 \%$. Regarding C-erbB-2 staining, the lack of staining on tumor cells or incomplete membrane staining $<10 \%$ was considered as 0 . Score 1 - weak and incomplete membrane staining $>10 \%$. Score 2 - weak to moderate complete membrane staining $>10 \%$. Score 3 - strong complete membrane staining $>10 \%{ }^{6}$ Subgroups were determined according to the WHO classification (Table 1). ${ }^{4}$

\section{Results}

All patients were female with a median age of 61 years (41-87 years). Two of them were premenopausal and $1 \mathrm{pa}-$ tient had 2 cases of breast cancer at her pedigree. Clinical and histological findings of the patients with metaplastic cancer are summarized in Table 2.
Table 1. WHO classification of metaplastic carcinoma of the breast ${ }^{2}$

\begin{tabular}{l|} 
Metaplastic carcinoma \\
low-grade adenosquamous carcinoma \\
Fibromatosis-like metaplastic carcinoma \\
squamous cell carcinoma \\
spindle cell carcinoma \\
carcinoma associated with mesenchymal differentiation \\
Chondroid differentiation \\
osseous differentiation \\
other types of mesenchymal differentiation \\
Myoepithelial carcinoma
\end{tabular}

Table 2. Clinical and histologic findings in patients with metaplastic cancer

\begin{tabular}{|l|c|}
\multicolumn{1}{|c|}{ Characteristics } & $\begin{array}{c}\text { Metaplastic breast cancer } \\
(\mathrm{n}=7)\end{array}$ \\
\hline Median age & $61(41-87)$ \\
\hline Tumor size & \\
T1 & 1 \\
T2 & 4 \\
T3 & - \\
T4 & 2 \\
\hline Multicentric & 2 \\
\hline Lymph node & 1 \\
N0 & 5 \\
N1 & 1 \\
N2 & \\
\hline Metastasis & 6 \\
M0 & 1 \\
M1 & 1 \\
\hline Stage & 1 \\
stage IIA & 1 \\
stage IIB & 1 \\
stage IIIB & \\
stage IV & 2 \\
\hline Dominant metaplastic component & 1 \\
epithelial type metaplasia & \\
squamous & \\
spindle & \\
mixed type metaplasia & \\
osseous & \\
\hline
\end{tabular}

Two patients had draining wounds due to the tumor. Five patients had tumors with diameters $<5 \mathrm{~cm}$. The tumors were multi-centric in 2 patients and were associated with foci of carcinoma in situ. All patients had palpable and multiple axillary lymph nodes. Lymph nodes formed a conglomerate in 1 patient. This patient also had lung, bone and brain metastases at diagnosis.

Two patients were diagnosed with stage IIIB and 1 patient had 3 cycles of neoadjuvant chemotherapy. Modified radical mastectomy was performed in the cases with stages IIA, IIB, and those that had neoadjuvant chemotherapy. Toilet mastectomy was performed in the case of stage IIIB with a draining wound. The patient with stage IV was diagnosed by a core biopsy, but was not operated.

The patient with metastatic disease refused chemotherapy. The 87-year-old patient with stage IV could not have any treatment due to her other health problems. 
Two patients did not keep their follow-up and no information could be obtained about their final status. Four patients had CA (doxorubicin and cyclophosphamide) chemotherapy regimen. Two of these patients had paclitaxel treatment for 12 weeks. The patient that was ER and PR positive had 4 cycles of CA and tamoxifen $(2 \times 10 \mathrm{mg})$ treatment later. A locally advanced patient had 3 cycles of neoadjuvant and 3 cycles of postsurgical CAF (cyclophosphamide, doxorubicin and 5-florourasil) treatment. The median follow-up duration of the patients was 34 (10-58) months. Three out of 5 followed-up cases were diseasefree, while 2 of them died due to metastases (Table 3).

\section{Histological and immunohistochemical analysis}

After the examination of the H\&E preparations, 4 patients were demonstrated to have a squamous type of metaplastic cell differentiations, 1 spindle, 1 adenosquamous, and 1 osseous. Six of 7 patients were found to be estrogen and progesterone receptors and C-erbB-2 expression negative. In 1 patient with squamous (adenosquamous) metaplasia, ER was $10 \%$ and PR was $5 \%$ positive. Conventional fluorescent in situ hybrilization (FISH) method did not show any HER2/neu gene amplification upon (+2) C-erB-2 expression score in the same patient. CK5/6 was positive in 5 patients. CK5/6 could not be evaluated in 1 patient despite consequent stains. It was negative in 1 patient. Epidermal growth factor receptor was positive in all patients (Table 4).

\section{Discussion}

DNA microarray and immunohistochemical methods have been quite popular recently in determining the subgroups of breast cancer biologically and clinically. Considering gene expressions, breast cancer that is ER (+) is classified into 2 subgroups as luminal A and luminal B, and ER (-) is classified into 3 subgroups as HER2 expressing, basal-like and null (similar to normal), although there have not been any certain criteria accepted yet. ${ }^{7}$ With this classification, we aim to obtain data that would present patient follow-up and treatment choice, demonstrating the molecular basis of the heterogeneity that is observed in breast cancer.

Triple-negative breast cancer (TNBC) is a heterogeneous tumor group that makes $10-20 \%$ of all breast cancers and has an aggressive clinical course. ${ }^{8,9} 55-80 \%$ of TNBC has a basaloid phenotype. ${ }^{8,10}$ Cytokeratin (CK) 14, CK5/6, CK 17,epithelial growth factor receptor (EGFR), p63, CD10, laminin, KIT, nestin, caveolin 1, and NGFR can be used as basal or myoepithelial cell determinant. Basal-like breast cancers show expressions of basal cytokeratin with a high molecular weight (CK5/6, CK14 and CK17), p-kadherin and fascin. In determining basal-like breast cancers, specificity of being ER (-), HER2 (-), CK5/6 and EGFR (+) is 100\%, and sensitivity is $76 \% .{ }^{11}$ In more than $90 \%$ of $\mathrm{MpBC}$, CK5/6, CK14 and EGFR expressions are positive, while ER, PR and HER2 are negative immunohistochemically. Estrogen receptor or PR expression frequency is $0-17 \%$ in MpBC..$^{5}$ All our cases are triple-negative, except the patient in the grey zone. Considering this, all the presented patients can be

Table 3. Follow-up (7 patients)

\begin{tabular}{|c|c|c|c|c|c|c|c|}
\hline Follow-up period & & & 58 months & 56 months & 34 months & 10 months & 11 months \\
\hline $\begin{array}{l}\text { Disease-free } \\
\text { survival }\end{array}$ & - & - & 58 months & 56 months & 34 months & $\begin{array}{l}\text { T4N1M0 } \\
\text { at diagnosis }\end{array}$ & metastatic \\
\hline Tamoxifen & - & - & + & - & - & - & - \\
\hline Chemotherapy & 4 cycles of CA & 4 cycles of CA & 4 cycles of CA & $\begin{array}{c}3+3 \text { cycles } \\
\left.\operatorname{CAF}^{*}\right)\end{array}$ & $\begin{array}{c}4 \text { cycles of CA + } \\
\text { Paclitaxel }\end{array}$ & Paclitaxel & $\begin{array}{l}\text { refused } \\
\text { treatment }\end{array}$ \\
\hline Survival & & & $\begin{array}{c}\text { alive, } \\
\text { disease-free }\end{array}$ & $\begin{array}{l}\text { alive, disease- } \\
\text { free }\end{array}$ & $\begin{array}{c}\text { alive, } \\
\text { disease-free }\end{array}$ & $\begin{array}{l}10 \text { months } \\
\text { metastatic }\end{array}$ & \\
\hline Differentiation & osseous & squamous & squamous (\#) & squamous & spindle & squamous & squamous \\
\hline
\end{tabular}

* 3 cycles of neoadjuvant, 3 cycles of adjuvant chemotherapy; \# adenosquamous type.

Table 4. Results of differentiation and immunohistochemical analyses

\begin{tabular}{|c|c|c|c|c|c|c|c|}
\hline & 1 & 2 & 3 & 4 & 5 & 6 & 7 \\
\hline & osseous & squamous & squamous $\left(^{*}\right)$ & squamous & spindle & squamous & squamous \\
\hline ER & - & - & $++/+++$ & - & - & - & - \\
\hline PR & - & - & $+/+++$ & - & - & - & - \\
\hline C-erbB-2 & - & - & $++/++++$ & - & - & - & - \\
\hline CK5/6 & - & + & $?$ & + & + & + & + \\
\hline EGFR & + & + & + & + & + & + & + \\
\hline
\end{tabular}

* adenosquamous type. 
accepted as TNBC. CK5/6 expression could not be evaluated in the case with adenosquamous differentiation, while it was negative in the case with osseous differentiation. $\mathrm{MpBC}$ can be evaluated as a subgroup of TNBC considering its immunohistochemical characteristics. Five of the patients presented with squamous differentiation resembled the subgroup of TNBC with a basaloid phenotype. Positivity of the EGFR expression in all cases of MpBC suggests that antiangiogenic treatment alternatives may be helpful. ${ }^{2}$

Immunohistochemically, $\mathrm{C}$-erbB-2 scoring is a subjective evaluation. $\mathrm{C}$-erbB- 2 score was +2 in 1 patient of the group presented. However, there was no HER2/neu gene amplification with the FISH method. ER and PR positivity were within the grey zone limits in this patient. The patient, whose cancer was classified as T1N1M0 (stage IIA), had 4 cycles of AC chemotherapy. The patient used tamoxifen $(2 \times 10 \mathrm{mg})$ for 4 years and is disease-free at present.

The incidence of $\mathrm{MpBC}$ within breast cancers is reported as $0.02-0.5 \%{ }^{1,2,12}$ It made $0.6 \%$ of the cases that had been diagnosed with breast cancer at our pathology unit during 12 years. Metaplastic cell differentiation with squamous cells was the most frequent. However, 1 patient had sarcomatous changes that showed osseous differentiation.

Metoplastic carcinoma of the breast is mostly seen in the $5^{\text {th }}$ decade. Compared to classical breast carcinoma, tumor size is bigger, axillary lymph node involvement is less frequent and axillary lymph node involvement at the diagnosis is $8-40 \%$ in MpBC. ${ }^{5,13-15}$ Six of the 7 patients presented had a metastatic lymph node. Metoplastic carcinoma of the breast is observed to metastasize to the bones and lungs with hematogeneous spread rather than lymphatic spread.

The majority of $\mathrm{MpBC}$ tumors is estrogen receptor (ER), progesterone receptor (PR), and HER2 negative, triple-negative breast cancer (TNBC), and usually carries a worse prognosis compared to non-metaplastic TNBC. ${ }^{3,5}$ Metoplastic carcinoma of the breast is usually more aggressive than pure invasive ductal or invasive lobular cancers often presenting with larger staging (T2, T3); however, lymph node involvement is less likely to be noted. ${ }^{2}$ The prognosis of $\mathrm{MpBC}$ was poorer than that of invasive ductal carcinoma and TNBC; the 5-year overall survival rate was 54.5\% in MpBC vs $85.1 \%$ in invasive ductal carcinoma and $73.3 \%$ in TNBC $(\mathrm{p}<0.001) .{ }^{16}$ In a multicentric study of 405 patients, it was differently reported that there was no difference between ductal breast cancers and $\mathrm{MpBC}$; however, spindle cell type shows aggressive biologic behavior and chemotherapy provides a longer survival in an early stage. ${ }^{17}$

Clinical findings are like in other breast cancers. MpBC can show benign characteristics at sonography, mammography and MRI as well as findings of invasive carcinoma. It has no specific radiologic findings. ${ }^{13,18}$ Radiologic findings can change according to the component the tumor contains. ${ }^{19}$ Metoplastic carcinoma of the breast is seen as a spiculated mass with irregular margins that often has intermediate to high signal intensity in T2W images and is low or isointense in T1W images in MRI. ${ }^{13}$
Metaplastic carcinoma of the breast may be treated with breast-conserving surgery in suitable cases. It is reported that there is no difference in the survival rate between breast-conserving surgery and a radical mastectomy. ${ }^{1,20}$ However, a modified radical mastectomy is the preferred method of treatment if the tumor is big at diagnosis and local recurrence rate is $35-62 \%$ in the first $2-5$ years compared to the recurrence rate of $17-20 \%$ for invasive ductal carcinoma of similar tumor size. ${ }^{1}$

There is no specific guideline for the treatment of $\mathrm{MpBC}$. It is known that long-term adjuvant chemotherapy is not useful in such cases. ${ }^{5,21}$ In 42 out of 47 cases of breast cancer, no survival benefit from adjuvant chemotherapy. ${ }^{5}$ The heterogeneous nature and sarcoma-like characteristics of MpBC are blamed for the failure of standard chemotherapy schemes. ${ }^{21}$ Today, for the treatment of MpBC, a high-dose anthracycline is suggested as a chemotherapy regimen in the case of epithelial subgroups. There is a need for new studies that would evaluate different choices of treatment as $\mathrm{MpBC}$ has a bad prognosis and an aggressive nature.

In conclusion, $\mathrm{MpBC}$ is relatively rare and, in our series, its incidence was $0.6 \%$. According to its immunohistochemical characteristics, $\mathrm{MpBC}$ can be interpreted as a subgroup of triple-negative breast cancers (TNBC). Most of the presented patients resembled the subgroup of TNBC with a basaloid phenotype.

\section{References}

1. Hu Q, Chen WX, Zhong SL, et al. Current progress in the treatment of metaplastic breast carcinoma. Asian Pac J Cancer Prev. 2013;14:6221-6225.

2. Abouharb S, Moulder S. Metaplastic breast cancer: Clinical overview and molecular aberrations for potential targeted therapy. Curr Oncol Rep. 2015;17(3):431.

3. Nelson RA, Guye ML, Luu T, Lai LL. Survival outcomes of metaplastic breast cancer patients: Results from a US population-based analysis. Ann Surg Oncol. 2015;22(1):24-31.

4. Reis-Filho JS, Lakhani SR, Gobbi H, Sneige N. Metaplastic carcinoma. In: Lakhani SR, Schnitt SJ, Tan PH, Vijner MJ, eds. WHO Classification of Tumours of the Breast. Lyon, France: IARC Pres: 2012;48-52.

5. Bae SY, Lee SK, Koo MY, et al. The prognoses of metaplastic breast cancer patients compared to those of triple-negative breast cancer patients. Breast Cancer Res Treat. 2011;126:471-478.

6. Rosai J. Surgical Pathology. $10^{\text {th }}$ ed. St. Louis, MO: Mosby Elsevier; 2011:1659-1770.

7. Sørlie T, Perou CM, Tibshirani R, et al. Gene expression patterns of breast carcinomas distinguish tumor subclasses with clinical implications. Proc Natl Acad Sci USA. 2011;98:10869-10874.

8. Bauer KR, Brown M, Cress RD, Parise CA, Caggiano V. Descriptive analysis of estrogen receptor (ER)-negative, progesterone receptor (PR)negative, and HER2-negative invasive breast cancer, the so-called triple-negative phenotype: A population-based study from the California Cancer Registry. Cancer. 2007;109:1721-1728.

9. Rakha EA, El-Sayed ME, Green AR, Lee AH, Robertson JF, Ellis IO. Prognostic markers in triple-negative breast cancer. Cancer. 2007;109:25-32.

10. Tischkowitz M, Brunet JS, Bégin LR, et al. Use of immunohistochemical markers can refine prognosis in triple-negative breast cancer. BMC Cancer. 2007;7:134.

11. Nielsen TO, Hsu FD, Jensen K, et al. Immunohistochemical and clinical characterization of the basal-like subtype of invasive breast carcinoma. Clin Cancer Res. 2004;10:5367-5374.

12. LaiHW, Tseng LM, Chang TW, et al. The prognostic significance of metaplastic carcinoma of the breast (MpBC): A case controlled comparison study with infiltrating ductal carcinoma. Breast. 2013;22:968-973. 
13. Choi BB, Shu KS. Metaplastic carcinoma of the breast: Multimodality imaging and histopathologic assessment. Acta Radiol. 2012;53:5-11.

14. Chao TC, Wang CS, Chen SC, Chen MF. Metaplastic carcinomas of the breast. J Surg Oncol. 1999;71:220-225.

15. Beatty JD, Atwood M, Tickman R, Reiner M. Metaplastic breast cancer: Clinical significance. Am J Surg. 2006;191:657-664.

16. Song $Y$, Liu X, Zhang G, et al. Unique clinicopathological features of metaplastic breast carcinoma compared with invasive ductal carcinoma and poor prognostic indicators. World J Surg Oncol. 2013;11:129.

17. Rakha EA, Tan PH, Varga Z, et al. Prognostic factors in metaplastic carcinoma of the breast: A multi-institutional study. Br J Cancer. 2015;112(2):283-289.

18. Günhan-Bilgen I, Memiş A, Ustün EE, Zekioglu O, Ozdemir N. Metaplastic carcinoma of the breast: Clinical, mammographic, and sonographic findings with histopathologic correlation. AJR Am J Roentgenol. 2002;178:1421-1425.

19. Greenberg D, Mclntyre H, Bierre T. Metaplastic breast cancer. Australas Radiol. 2004;48:243-247.

20. Dave G, Cosmatos H, Do T, Lodin K. Varshney D. Metaplastic carcinoma of the breast: A retrospective review. Int J Radiat Oncol Biol Phys. 2006;64:771-775.

21. Al Sayed AD, El Weshi AN, Tulbah AM, Rahal MM, Ezzat AA. Metaplastic carcinoma of the breast clinical presentation, treatment results and prognostic factors. Acta Oncol. 2006;45:188-195. 